\title{
ORIGINAL
}

\section{Mechanical power normalized to predicted body weight as a predictor of mortality in patients with acute respiratory distress syndrome}

\author{
Zhongheng Zhang ${ }^{1 *} \mathbb{0}$, Bin Zheng ${ }^{2}$, Nan Liu ${ }^{3,4}$, Huiqing $\mathrm{Ge}^{5}$ and Yucai Hong ${ }^{1}$
}

(c) 2019 Springer-Verlag GmbH Germany, part of Springer Nature

\begin{abstract}
Purpose: Protective mechanical ventilation based on multiple ventilator parameters such as tidal volume, plateau pressure, and driving pressure has been widely used in acute respiratory distress syndrome (ARDS). More recently, mechanical power (MP) was found to be associated with mortality. The study aimed to investigate whether MP normalized to predicted body weight (norMP) was superior to other ventilator variables and to prove that the discrimination power cannot be further improved with a sophisticated machine learning method.

Methods: The study included individual patient data from eight randomized controlled trials conducted by the ARDSNet. The data was split 3:1 into training and testing subsamples. The discrimination of each ventilator variable was calculated in the testing subsample using the area under receiver operating characteristic curve. The gradient boosting machine was used to examine whether the discrimination could be further improved.

Results: A total of 5159 patients with acute onset ARDS were included for analysis. The discrimination of norMP in predicting mortality was significantly better than the absolute MP ( $p=0.011$ for DeLong's test). The gradient boosting machine was not able to improve the discrimination as compared to norMP ( $p=0.913$ for DeLong's test). The multivariable regression model showed a significant interaction between norMP and ARDS severity $(p<0.05)$. While the norMP was not significantly associated with mortality outcome (OR 0.99; $95 \% \mathrm{Cl} 0.91-1.07 ; p=0.862$ ) in patients with mild ARDS, it was associated with increased risk of mortality in moderate (OR $1.11 ; 95 \% \mathrm{Cl} 1.02-1.23 ; p=0.021)$ and severe (OR 1.13; 95\% Cl 1.03-1.24; $p<0.008)$ ARDS.

Conclusions: The study showed that norMP was a good ventilator variable associated with mortality, and its predictive discrimination cannot be further improved with a sophisticated machine learning method. Further experimental trials are needed to investigate whether adjusting ventilator variables according to norMP will significantly improve clinical outcomes.
\end{abstract}

Keywords: Acute respiratory distress syndrome, Mortality, Gradient boosting machine, Mechanical power

\footnotetext{
*Correspondence: zh_zhang1984@zju.edu.cn

${ }^{1}$ Department of Emergency Medicine, Sir Run Run Shaw Hospital,

Zhejiang University School of Medicine, No 3, East Qingchun Road, Hangzhou 310016, Zhejiang, China

Full author information is available at the end of the article
}

\section{实 Springer}




\section{Introduction}

Mechanical ventilation is strongly recommended for ARDS patients to avert life-threatening hypoxia and hypercapnia in respiratory failure; however, it is also associated with ventilator-induced lung injury (VILI) [1, 2]. There was experimental evidence showing that VILI was influenced by every aspect of ventilator settings. For example, the severity of VILI was demonstrated to be dependent on respiratory rate at a given level of tidal volume [3]. Thus, we needed to monitor a number of key parameters to ensure that mechanical ventilation do not lead to VILI, such as low tidal volume, high positive end-expiratory pressure (PEEP), limited plateau pressure and driving pressure [4-7]. Mechanical power (MP), as calculated by the combination of tidal volume, PEEP, plateau pressure, peak inspiratory pressure (PIP), and respiratory rate, was proposed to better capture the total energy delivered to the lung parenchyma $[8,9]$. Since MP integrated many aspects of mechanical ventilation, it is theoretically superior to each of the individual ventilator variables. There was empirical evidence showing that MP was able to predict risk of mortality in mechanically ventilated patients [9]. However, we are not sure whether MP can better stratify risk than other individual ventilator variables. The first aim of the study is to empirically compare the discrimination power of using MP in predicting ARDS mortality versus using other individual ventilator variables. We hypothesized that MP-based prediction can be significantly better than other ventilator variables.

Furthermore, we know that the effect of MP might be influenced by the functional lung size. In other words, the discrimination power of MP normalized to lung size would theoretically be improved as compared with the absolute MP value [10]. This is in line with the fact that tidal volume when normalized to predicted body weight (PBW) showed greater accuracy than the absolute value [11]. Our second goal in this paper was to investigate whether the discrimination power of MP normalized to PBW was better than the absolute value of MP. Additionally, the functional lung size is not always proportional to the PBW in ARDS patients. For example, the severely injured lung might have smaller functional lung size regardless of the body height of the patient. Thus, we needed to take the severity of ARDS into the consideration when we examined the association of MP with mortality. Our third hypothesis was that the MP is more harmful in severe ARDS (e.g., severe ARDS has smaller functional lung size than mild ARDS) than mild ones.

$M P$ is a ventilator parameter with concrete meaning in physics. However, the mathematical equation for computing MP may not fully capture the predictive information from ventilator variables. Thus, we intended to

\section{Take-home message}

Mechanical power normalized to body weight (norMP) showed signifcantly greater diagnostic performance in predicting mortality than absolute value of mechanical power, tidal volume, plateau pressure, and driving pressure.

The predictive performance of norMP cannot be further improved by using sophisticated machine learning techniques.

The impact of norMP on mortality outcome was dependent on the severity of ARDS. While the norMP was not significantly associated with mortality outcome in patients with mild ARDS, it was associated with increased risk of mortality in moderate and severe ARDS.

norMP is a potential biomarker for monitoring ventilator-induced lung injury.

explore whether the predictive power could be further improved with the same ventilator variables by using the gradient boosting machine (GBM). GBM has been shown to be able to approximate nearly any functional form by increasing the number of boosting iterations [12, 13]. If the GBM could not further improve the predictive power, MP would be considered as the near-optimal ventilator variable to monitor potential VILI. Many modern machine learning algorithms can identify high-order interactions among variables, while minimizing the risk of overfitting.

\section{Methods}

\section{Data source}

The study included ARDS patient data from eight randomized controlled trials (RCTs) conducted by the ARDSNet $[5,7,14-21]$. The LaSRS trial was excluded from analysis because it enrolled patients with stable or worsening ARDS for 7-28 days and was simply not comparable to all the other early ARDS intervention trials. All patients included for these trials were analyzed. All individual RCTs were approved by the ethics committee of participating centers and informed consent was obtained. The data were available in the Biologic Specimen and Data Repository Information Coordinating Center (https ://biolincc.nhlbi.nih.gov/). The secondary analyses of the data were approved by the ethics committee of the Sir Run Run Shaw Hospital.

\section{Variables}

All crude data used in our study were extracted from the original trials. Patient demographics such as Acute Physiology and Chronic Health Evaluation (APACHE) III, age, gender, type of ICU, and admission source were extracted from the case report form. Baseline (day 0) ventilator parameters were defined as the most recent values prior to randomization. Corrected inspired tidal volume $\left(V_{\mathrm{T}}\right)$ as recorded in original trials was 
used for current analysis. Positive end-expiratory pressure (PEEP) was the external or applied PEEP, not the total PEEP, auto-PEEP, or intrinsic PEEP. The plateau pressure $\left(P_{\text {plateau }}\right)$ was measured with a 0.5 -s inspiratory pause. Peak inspiratory pressure (PIP) should be obtained while the patient is relaxed, not coughing or moving in bed. Mechanical power was computed by the following equation $[8,9]$ :

$$
\mathrm{MP}(\mathrm{J} / \mathrm{min})=0.098 \times V_{\mathrm{T}} \times \mathrm{RR} \times(\mathrm{PIP}-\Delta P \times 0.5),
$$

where the driving pressure $\Delta P=P_{\text {plateau }}-$ PEEP.

$$
\operatorname{norMP}\left(\times 10^{-3} \mathrm{~J} / \mathrm{min} / \mathrm{kg}\right)=\mathrm{MP} / \mathrm{PBW},
$$

where $\mathrm{PBW}$ was the predicted body weight measured in kilograms.

Lung compliance was computed by the following equation:

$$
\text { Compliance }=V_{\mathrm{T}} /\left(P_{\text {plateau }}-\mathrm{PEEP}\right) .
$$

Then MP could be normalized by the compliance:

$$
\text { MP normalized to compliance }=\frac{\text { MP }}{\text { Compliance }} \text {. }
$$

Missing data [electronic supplemental material (ESM) Fig. 1] were handled with a multiple imputation method [22].

\section{Clinical outcome}

The primary outcome of the study was the mortality rate at 90 days. A patient is defined as alive if he/she is at home with unassisted breathing at any time up through day 90 . Home is defined as the place the patient lived prior to study hospital admission. A patient was defined as expired if he/she died before being discharged from the hospital and staying at home with unassisted breathing or died prior to achieving unassisted breathing at home for $48 \mathrm{~h}$.

\section{Comparing ventilator parameters}

Each of the ventilator variables including $V_{\mathrm{T}}, V_{\mathrm{T}}$ normalized to predicted body weight, respiratory rate, PIP, $\Delta P$, MP, $P_{\text {plateau }}$ PEEP, norMP, and MP normalized to compliance were entered into logistic regression models, resulting in a total of ten models being trained. The whole sample was split 3:1 into training and testing subsamples. The discrimination of each model was calculated in the testing subsample using the area under receiver operating characteristic curve (AUROC). The difference of AUROC was tested by using Delong's method [23].

\section{Gradient boosting machine}

GBM is able to produce a prediction model in the form of an ensemble of weak prediction models (decision trees in our study). It builds the model in a stage-wise fashion and it generalizes them by allowing optimization of an arbitrary differentiable loss function. The feature space of the GBM comprised ventilator variables used for computing MP including $V_{\mathrm{T}}, \Delta P, P_{\text {plateau }}$, PEEP, PIP, and respiratory rate. The advantage of GBM is that it can approximate any functional form between mortality outcome and ventilator variables. Again, the GBM was trained on the training subsample and tested on the testing subsample. Hyperparameter tuning was implemented with the tenfold cross-validation method. A tuning parameter grid was used by varying the number of trees (number of boosting iterations) from 10 to 300 and the maximum depth of each tree from $1,3,5$, and 7 . The learning rate or step-size reduction was set to 0.1 . The minimum number of observations in the terminal nodes of the trees was 20. Gradient boosting was performed by using the caret package (v6.0-81) in R.

\section{Effect of MP on mortality by ARDS severity}

The interaction between MP and ARDS severity was explored by a multivariable logistic regression model, adjusting for potential confounders such as age, gender, APACHE III, admission source, and ICU type. ARDS severity was described by using the Berlin definition, where the mild, moderate, and severe ARDS were categorized at the cutoff $P / F$ ratio of 100 and $200 \mathrm{mmHg}$ [24]. However, we could not make sure that every criterion of the Berlin definition was met. We further investigated the association of norMP with risk of worsening in patients with mild ARDS at day 0 . Worsening ARDS was defined as when moderate or severe ARDS criteria were met after day 2 [25].

All statistical analyses mentioned above were performed using RStudio (Version 1.1.463).

\section{Results}

\section{Characteristics of included trials}

A total of 5159 patients with acute onset ARDS were included for analysis. Characteristics of patients and ventilator variables across the ARDSNet trials are shown in ESM Table 1. The FACCT study $(n=1000)$ had the largest sample size and the OMEGA trial $(n=272)$ included the smallest number of patients. While patients in the ALVEOLI trial had the highest APACHE III score (96.7 \pm 30.3$)$, the ARDSnet1 had the lowest APACHE III score $(85.1 \pm 24.8)$. On day 0 , tidal volume was the smallest in the OMEGA trial $(430.5 \pm 90.1 \mathrm{~mL})$, and was the highest in the ARDSnet1 trial $(669.6 \pm 120.2 \mathrm{~mL})$. The 
MP was also the highest in the ARDSnet1 trial [29.6 $(21.3,41.1) \mathrm{J} / \mathrm{min}]$, and was the lowest in SAILS trial [19.2 (13.6, 26.3) $\mathrm{J} / \mathrm{min}]$. The SAILS $\left(347.7 \pm 179.5 \times 10^{-3} \mathrm{~J} /\right.$ $\mathrm{min} / \mathrm{kg})$ and OMEGA $\left(352.3 \pm 190.3 \times 10^{-3} \mathrm{~J} / \mathrm{min} / \mathrm{kg}\right)$ trials reported the lowest norMP versus the ARDSnet1 trial $\left(512.9 \pm 264.8 \times 10^{-3} \mathrm{~J} / \mathrm{min} / \mathrm{kg}\right)$.

\section{Predictive performance of ventilator variables}

Each of the ventilator variables was regressed on mortality in the training set and AUROC of each model was computed in the testing set (Table 1). The norMP showed the highest AUROC among all ventilator variables. The discrimination of norMP was significantly better than the absolute MP ( $p=0.011$ for DeLong's test). The gradient boosting machine was not able to improve the discrimination as compared to the MP ( $p=0.973$ for DeLong's test) or norMP ( $p=0.913$ for DeLong's test). MP normalized to compliance was among the variables with the highest predictive discrimination (AUC 0.753; 95\% CI

Table 1 Discrimination of ventilator parameters measured on day $\mathbf{0}$ in predicting mortality outcome

\begin{tabular}{|c|c|c|c|}
\hline Ventilator parameters & AUROC & $\begin{array}{l}\text { Lower } \\
\text { limit } \\
\text { of } 95 \% \mathrm{Cl}\end{array}$ & $\begin{array}{l}\text { Upper } \\
\text { limit } \\
\text { of } 95 \% \mathrm{CI}\end{array}$ \\
\hline Tidal volume (mL) & 0.744 & 0.713 & 0.775 \\
\hline $\begin{array}{l}\text { Tidal volume normalized to PBW } \\
(\mathrm{mL} / \mathrm{kg})\end{array}$ & 0.746 & 0.715 & 0.777 \\
\hline Respiratory rate (/min) & 0.743 & 0.713 & 0.774 \\
\hline PEEP $\left(\mathrm{cmH}_{2} \mathrm{O}\right)$ & 0.744 & 0.713 & 0.775 \\
\hline Plateau pressure $\left(\mathrm{cmH}_{2} \mathrm{O}\right)$ & 0.747 & 0.716 & 0.778 \\
\hline $\mathrm{PIP}\left(\mathrm{cmH}_{2} \mathrm{O}\right)$ & 0.746 & 0.715 & 0.777 \\
\hline Driving pressure $\left(\mathrm{cmH}_{2} \mathrm{O}\right)$ & 0.743 & 0.712 & 0.774 \\
\hline $\mathrm{MP}(\mathrm{J} / \mathrm{min})$ & 0.747 & 0.717 & 0.778 \\
\hline norMP $\left(10^{-3} \mathrm{~J} / \mathrm{min} / \mathrm{kg}\right)$ & 0.751 & 0.720 & 0.781 \\
\hline MP normalized to compliance & 0.753 & 0.722 & 0.783 \\
\hline Gradient boosting machine & 0.748 & 0.717 & 0.779 \\
\hline
\end{tabular}

All logistic regression models included APACHE III, age, gender, and $P / F$ ratio plus each of the individual ventilator parameters in a training data set and validated in a testing data set. The whole sample was split into training and testing sets in the ratio of 3:1. The gradient boosting model was trained by incorporating APACHE III, age, gender, and $P / F$ ratio plus ventilator parameters of tidal volume, PEEP, PIP, plateau pressure, respiratory rate, and PBW. Hyperparameter tuning was performed in the training subsample with repeated cross-validation. The discriminations (AUROCs) of all variables or models were validated in the testing data set

The discrimination of norMP was also significantly better than the absolute MP ( $p=0.011$ for DeLong's test) and tidal volume ( $p=0.047$ for DeLong's test). The gradient boosting machine was not able to improve the discrimination as compared to the MP ( $p=0.973$ for DeLong's test) or normalized MP ( $p=0.913$ for DeLong's test). MP normalized to predicted body weight was similar to that normalized to lung compliance $(p=0.659)$

PEEP positive end-expiratory pressure, $P I P$ peak inspiratory pressure, norMP mechanical power normalized to predicted body weight, $M P$ mechanical power, $A U R O C$ area under receiver operating characteristic curve, $\mathrm{Cl}$ confidence interval, PBW predicted body weight
0.722-0.783), and it was not significantly different from the norMP $(p=0.659)$.

Sensitivity analysis by excluding patients with pressure control ventilation showed that norMP or MP normalized to compliance had higher discrimination than other ventilator variables such as tidal volume and driving pressure. The GBM was not able to significantly increase the discrimination (ESM Table 2).

\section{Multivariable regression model including an interaction term between norMP and ARDS severity}

A multivariable regression model including interaction between norMP and ARDS severity was trained on the whole data set (Table 2). The results showed that there was significant interaction between norMP and ARDS. While the norMP was not significantly associated with mortality outcome (OR 0.99; 95\% CI 0.91-1.07; $p=0.862$ ) in patients with mild ARDS, it was associated with increased risk of mortality in moderate (OR 1.11; 95\% CI 1.02-1.23; $p=0.021$ ) and severe (OR 1.13; 95\% CI $1.03-1.24 ; p=0.008$ ) ARDS (Fig. 1). The results indicated that the negative impact of higher norMP was dependent on the severity of ARDS. By restricting our analysis to mild ARDS at day 0, norMP was significantly associated with increased risk of subsequent worsening (OR for each 100 -unit increase in $\times 10^{-3} \mathrm{~J} / \mathrm{min} / \mathrm{kg}, 1.09 ; 95 \% \mathrm{CI}$ $1.02-1.17 ; p=0.019$; Table 3 ).

\section{Gradient boosting machine}

A tree-based gradient boosting machine was trained on the same ventilator variables used for computing norMP. The training process showed that the prediction accuracy was not significantly improved with increasing tree depth and the number of boosting iterations (Fig. 2). The AUROC of the best GBM model was not significantly different from the norMP $(0.748 ; 95 \%$ CI $0.717-0.779$ versus 0.750; 95\% CI 0.720-0781; $p=0.973$ for DeLong's test).

\section{Discussion}

The results of our study provide evidence to support our hypotheses. First, among all ventilator variables, norMP displayed a significantly higher AUROC value than any other individual variables including MP. Second, the effect of norMP was dependent on the severity of ARDS. Although norMP had no significant effect on mild ARDS patients with respect to the mortality outcome, norMP was associated with significantly increased risk of worsening of ARDS. This result supports the hypothesis that the effect of MP on VILI was dependent on the functional lung size. However, we must acknowledge that ARDS severity defined in the Berlin definition was not without critics and functional lung size and $P / F$ ratio may not correlate well in some cases [26]. Third, our study 
Table 2 Multivariable regression model with interaction between ARDS severity and mechanical power

\begin{tabular}{|c|c|c|}
\hline Variables & OR $(95 \% \mathrm{Cl})$ & $p$ value \\
\hline APACHE III (with each 10-point increase) & $1.25(1.22,1.28)$ & $<0.001$ \\
\hline Gender (female as reference) & $1.21(1.05,1.39)$ & 0.008 \\
\hline Age (with each 10-year increase) & $1.39(1.33,1.46)$ & $<0.001$ \\
\hline \multicolumn{3}{|l|}{ ICU type (burn as reference) } \\
\hline Cardiac SICU & $0.90(0.40,2.05)$ & 0.801 \\
\hline CCU & $1.04(0.52,2.14)$ & 0.912 \\
\hline MICU & $1.06(0.60,1.97)$ & 0.841 \\
\hline MICU/SICU & $0.78(0.43,1.47)$ & 0.414 \\
\hline Neuro ICU & $0.88(0.38,2.01)$ & 0.753 \\
\hline Other & $1.22(0.56,2.71)$ & 0.619 \\
\hline SICU & $0.97(0.53,1.85)$ & 0.911 \\
\hline Trauma & $0.75(0.40,1.48)$ & 0.393 \\
\hline \multicolumn{3}{|l|}{ Admission source (another hospital as reference) } \\
\hline Another special care unit & $1.86(1.23,2.79)$ & 0.003 \\
\hline Direct admission & $1.30(0.69,2.38)$ & 0.399 \\
\hline ER & $1.05(0.86,1.28)$ & 0.658 \\
\hline Floor & $1.76(1.44,2.17)$ & $<0.001$ \\
\hline OR & $0.91(0.64,1.29)$ & 0.604 \\
\hline Recovery room & $0.69(0.36,1.24)$ & 0.228 \\
\hline Stepdown unit & $1.73(1.21,2.46)$ & 0.002 \\
\hline Fluid balance on day 0 (with each 1-L increase) & $0.99(0.97,1.01)$ & 0.332 \\
\hline norMP (with each 100-unit increase in $\times 10^{-3} \mathrm{~J} / \mathrm{min} / \mathrm{kg}$ ) & $0.99(0.91,1.07)$ & 0.862 \\
\hline \multicolumn{3}{|l|}{ ARDS severity (mild as reference) } \\
\hline Severe & $1.37(1.10,1.70)$ & 0.005 \\
\hline Moderate & $1.28(1.05,1.58)$ & 0.017 \\
\hline \multicolumn{3}{|l|}{ Interaction between norMP and ARDS severity } \\
\hline norMP: severe & $1.13(1.03,1.24)$ & 0.008 \\
\hline norMP: moderate & $1.11(1.02,1.23)$ & 0.021 \\
\hline
\end{tabular}

ICU intensive care unit, MICU medical ICU, SICU surgical ICU, CCU coronary care unit, ER emergency room, OR operating room, MP mechanical power, ARDS acute respiratory distress syndrome, GBM gradient boosting model

employed GBM to explore whether there was additional information that can be extracted from simple ventilator variables to predict mortality. The result showed that GBM was not better than norMP in predicting mortality outcome, suggesting that norMP had fully captured predictive information from ventilator variables.

Individual ventilator variables have been widely investigated in previous studies. Early studies showed that low tidal volume ventilation is not only beneficial for ARDS patients but also for those with healthy lungs [5, 27-29]. In fact, low tidal volume is a component of protective ventilation and other components include PEEP, plateau pressure, respiratory rate, and peak inspiratory pressure $[6,7,30-32]$. Driving pressure $(\Delta P)$, which is computed by subtracting PEEP from plateau pressure, has received more attention in recent years because it considers lung compliance. There is a large body of evidence showing that lower driving pressure is associated with improved outcome in both injured and non-injured lungs $[6,10,33,34]$. In our study, we found that the discrimination of driving pressure in predicting mortality was slightly lower than that of norMP. This result suggests that there are residuals not captured by the $\Delta P$. For example, the respiratory rate is not considered in $\Delta P$, but it is an important determinant of VILI $[3,35]$. The PIP is related to the inspiratory flow, which may contribute to the locally intensified concentration of stress, a problem influenced by viscoelastic tissue properties. There is evidence that for a given plateau pressure, the rate at which the volume is given plays an important role in the genesis of VILI [36, 37]. Again, the driving pressure did not consider this component.

In contrast to conventional ventilator variables such as tidal volume, plateau pressure, driving pressure, and PEEP, MP consists of all aspects of mechanical ventilation with the assumption that VILI can be best predicted by the MP imposed on lung parenchyma. In previous studies, MP has been investigated for its association 


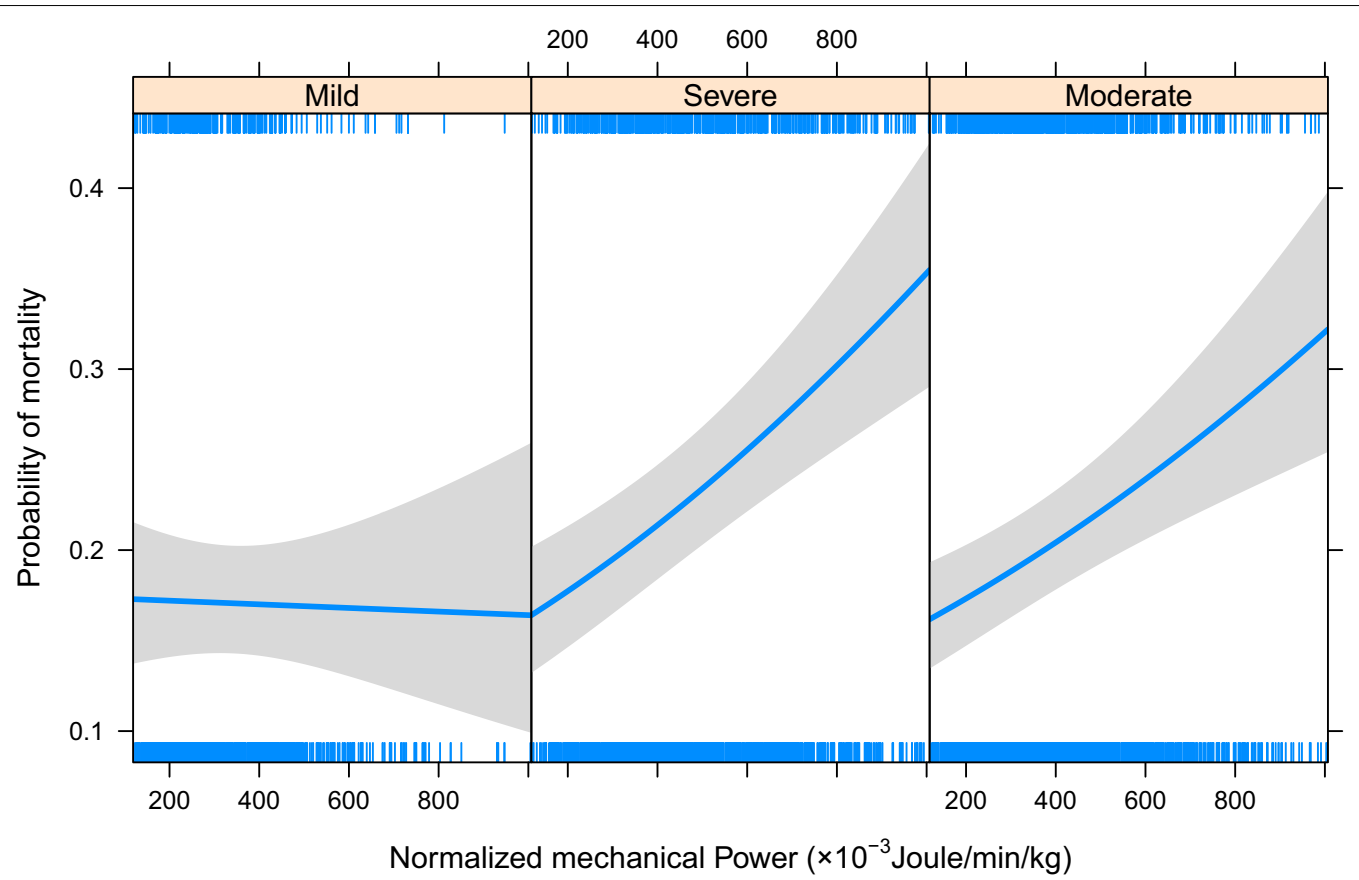

Fig. 1 Interaction between norMP and ARDS severity. While the norMP was not significantly associated with mortality outcome (OR $0.99 ; 95 \% \mathrm{Cl}$ $0.91-1.07 ; p=0.862)$ in patients with mild ARDS, it was associated with increased risk of mortality in moderate $(\mathrm{OR} 1.11 ; 95 \% \mathrm{Cl} 1.02-1.23 ; p=0.021)$ and severe (OR 1.13; 95\% Cl 1.03-1.24; $p=0.008)$ ARDS. norMP mechanical power normalized to predicted body weight, ARDS acute respiratory distress syndrome, OR odds ratio

Table 3 Multivariable logistic regression model investigating independent risk factors for worsening ARDS

\begin{tabular}{|c|c|c|}
\hline Variables & OR $(95 \% \mathrm{Cl})$ & $p$ value \\
\hline APACHE III (with each 10-point increase) & $0.98(0.93,1.04)$ & 0.520 \\
\hline Gender (female as reference) & $0.96(0.73,1.26)$ & 0.777 \\
\hline Age (with each 10-year increase) & $0.98(0.90,1.06)$ & 0.622 \\
\hline \multicolumn{3}{|l|}{ ICU type (burn as reference) } \\
\hline Cardiac SICU & $0.81(0.08,7.93)$ & 0.850 \\
\hline CCU & $1.49(0.46,4.86)$ & 0.502 \\
\hline MICU & $1.43(0.58,3.38)$ & 0.424 \\
\hline MICU/SICU & $1.77(0.70,4.34)$ & 0.216 \\
\hline Neuro ICU & $0.41(0.10,1.62)$ & 0.213 \\
\hline Other & $1.16(0.34,4.05)$ & 0.814 \\
\hline SICU & $1.47(0.55,3.79)$ & 0.431 \\
\hline Trauma & $0.45(0.16,1.21)$ & 0.117 \\
\hline \multicolumn{3}{|l|}{ Admission source (another hospital as reference) } \\
\hline Another special care unit & $0.59(0.25,1.43)$ & 0.226 \\
\hline Direct admit & $0.50(0.21,1.24)$ & 0.123 \\
\hline ER & $0.67(0.45,0.99)$ & 0.048 \\
\hline Floor & $0.75(0.48,1.16)$ & 0.201 \\
\hline OR & $0.53(0.27,1.02)$ & 0.055 \\
\hline Recovery room & $0.24(0.10,0.61)$ & 0.003 \\
\hline Stepdown unit & $0.37(0.18,0.77)$ & 0.008 \\
\hline norMP (with each 100-unit increase in $\times 10^{-3} \mathrm{~J} / \mathrm{min} / \mathrm{kg}$ ) & $1.09(1.02,1.17)$ & 0.019 \\
\hline
\end{tabular}

The study population was patients with mild ARDS on day 0 . The worsening ARDS was defined when moderate or severe acute respiratory distress syndrome criteria were met after day 2

ICU intensive care unit, MICU medical ICU, SICU surgical ICU, CCU coronary care unit, ER emergency room, OR operating room, MP mechanical power, ARDS acute respiratory distress syndrome, GBM gradient boosting model 


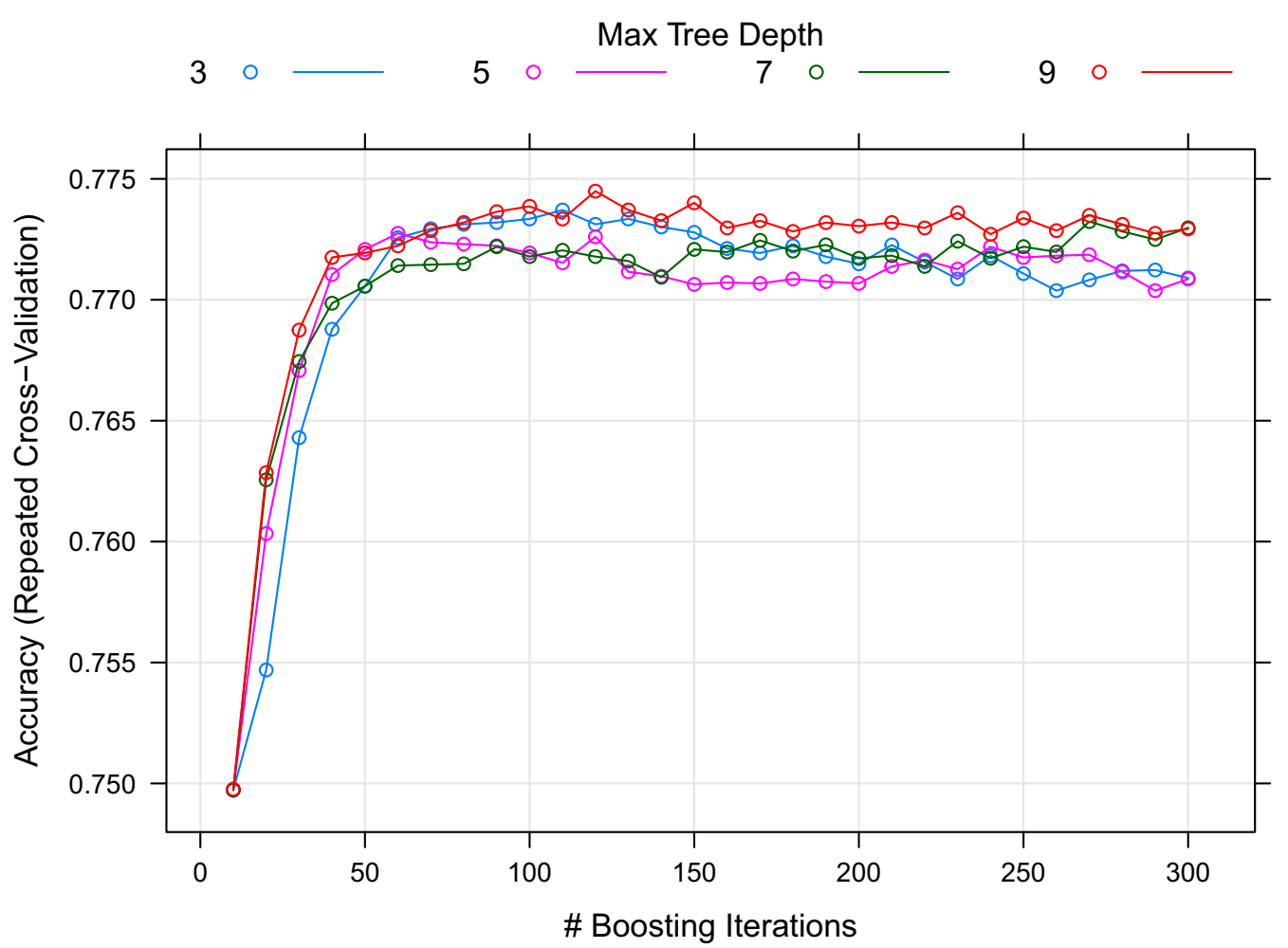

Fig. 2 The training process of the gradient boosting machine. The four lines represent trees with different complexities as represented by the maximum tree depth. It appears that a maximum depth of 9 (the maximum number of edges from the node to the tree's root node) can give the highest accuracy. However, the accuracy reaches a plateau at 50 boosting iterations

with mortality and the result found that MP was a strong predictor of in-hospital mortality (OR for each $5 \mathrm{~J} / \mathrm{min}$ increase in MP, 1.06; 95\% CI 1.01-1.11) [9]. More importantly, the result was robust even in patients receiving low tidal volume, indicating that tidal volume cannot fully explain the mortality outcome, and the residual can be captured by MP. However, that study did not directly compare the MP with other ventilator variables. Our study moved one step forward by providing evidence suggesting that the MP was better than other individual ventilator variables, and the discrimination power can be further enhanced by normalizing MP to the PBW or compliance.

Several limitations must be acknowledged. The first limitation came from clinical measurement on VILI. In routine clinical practice, we cannot directly quantify the occurrence of VILI in patients with ARDS. We used mortality rate in this study because VILI was closely linked to the mortality rate. The difficulty in quantifying the functional lung size was the second limitation of this study. At present, we can only indirectly describe the functional lung size by the PBW and ARDS severity. In the quantification of the severity of ARDS, the degree of hypoxemia is found to be predictive of mortality, while other candidate variables such as radiographic severity, respiratory system compliance $\left(\leq 40 \mathrm{~mL} / \mathrm{cmH}_{2} \mathrm{O}\right)$, PEEP $\left(\geq 10 \mathrm{cmH}_{2} \mathrm{O}\right)$, and corrected expired volume per minute $(\geq 10 \mathrm{~L} / \mathrm{min})$ were not associated with mortality [24]. Thus, our study employed the degree of hypoxemia to describe the homogeneity of the injured lungs. The third limitation was that we employed ventilator variables on day 0 in ARDSNet trials for predicting the mortality. In effect, the subsequent ventilator settings can have a significant impact on mortality outcome. However, ventilator variables on day 0 can better reflect real clinical practice and provide a wide distribution of each variable in the feature space. Further studies considering temporal changes of ventilator settings may be useful to confirm the value of norMP. Finally, the study was conducted in patients with injured lungs, and it is not known whether the result can be generalized to patients with healthy lungs but requiring mechanical ventilation. Therapeutic interventions in original trials may be confounding factors in our analysis. However, all trials except for the ARDSnet1 reported a neural effect of the intervention versus the control [38]. Since the ARDSnet1 trial compared low versus conventional tidal volume, and the comparators had been included in our analysis, the 
confounding induced by therapeutic interventions in the ARDSnet1 trial can be minimized.

In conclusion, we proved that norMP was a good ventilator variable in predicting mortality in ARDS patients. The predictive discrimination of norMP cannot be further improved with a sophisticated machine learning method, which means that norMP would be considered as the near-optimal ventilator variable to monitor potential VILI. Further studies are needed to investigate whether adjusting ventilator variables according to norMP will significantly improve clinical outcomes.

\section{Electronic supplementary material}

The online version of this article (https://doi.org/10.1007/s00134-019-05627-9) contains supplementary material, which is available to authorized users.

\begin{abstract}
Author details
${ }^{1}$ Department of Emergency Medicine, Sir Run Run Shaw Hospital, Zhejiang University School of Medicine, No 3, East Qingchun Road, Hangzhou 310016, Zhejiang, China. ${ }^{2}$ Department of Surgery, 2D, Walter C Mackenzie Health Sciences Centre, University of Alberta, Edmonton, AB, Canada. ${ }^{3}$ Duke-NUS Medical School, National University of Singapore, Singapore, Singapore. ${ }^{4}$ Health Services Research Centre, Singapore Health Services, Singapore, Singapore. ${ }^{5}$ Department of Respiratory Care, Sir Run Run Shaw Hospital, Zhejiang University School of Medicine, Hangzhou, China.
\end{abstract}

\section{Acknowledgements}

We would like to thank Leo M. A. Heunks for reviewing this manuscript and providing insightful comments.

\section{Funding}

Z. Z. received funding from Zhejiang Province Public Welfare Technology Application Research Project (CN) (LGF18H150005) and Scientific research project of Zhejiang Education Commission (Y201737841).

\section{Compliance with ethical standards}

\section{Conflicts of interest}

There is no conflict of interest.

\section{Publisher's Note}

Springer Nature remains neutral with regard to jurisdictional claims in published maps and institutional affiliations.

Received: 14 February 2019 Accepted: 22 April 2019

Published online: 6 May 2019

\section{References}

1. Herasevich V, Tsapenko M, Kojicic M et al (2011) Limiting ventilatorinduced lung injury through individual electronic medical record surveillance. Crit Care Med 39:34-39. https://doi.org/10.1097/CCM.0b013e3181 fa4184

2. Gattinoni L, Marini JJ, Collino F et al (2017) The future of mechanical ventilation: lessons from the present and the past. Crit Care 21:183. https ://doi.org/10.1186/s13054-017-1750-x

3. Cressoni M, Gotti M, Chiurazzi C et al (2016) Mechanical power and development of ventilator-induced lung injury. Anesthesiology 124:11001108. https://doi.org/10.1097/ALN.0000000000001056

4. Briel M, Meade M, Mercat A et al (2010) Higher vs lower positive end-expiratory pressure in patients with acute lung injury and acute respiratory distress syndrome: systematic review and meta-analysis. JAMA 303:865-873. https://doi.org/10.1001/jama.2010.218

5. Network Acute Respiratory Distress Syndrome, Brower RG, Matthay MA et al (2000) Ventilation with lower tidal volumes as compared with traditional tidal volumes for acute lung injury and the acute respiratory distress syndrome. N Engl J Med 342:1301-1308. https://doi. org/10.1056/NEJM200005043421801

6. Amato MBP, Meade MO, Slutsky AS et al (2015) Driving pressure and survival in the acute respiratory distress syndrome. N Engl J Med 372:747-755. https://doi.org/10.1056/NEJMsa1410639

7. Brower RG, Lanken PN, Maclntyre N et al (2004) Higher versus lower positive end-expiratory pressures in patients with the acute respiratory distress syndrome. N Engl J Med 351:327-336. https://doi.org/10.1056/ NEJMoa032193

8. Gattinoni L, Tonetti T, Cressoni M et al (2016) Ventilator-related causes of lung injury: the mechanical power. Intensive Care Med 42:15671575. https://doi.org/10.1007/s00134-016-4505-2

9. Serpa Neto A, Deliberato RO, Johnson AEW et al (2018) Mechanical power of ventilation is associated with mortality in critically ill patients: an analysis of patients in two observational cohorts. Intensive Care Med 44:1914-1922. https://doi.org/10.1007/s00134-018-5375-6

10. Tonetti T, Vasques F, Rapetti F et al (2017) Driving pressure and mechanical power: new targets for VILI prevention. Ann Transl Med 5:286. https://doi.org/10.21037/atm.2017.07.08

11. Linares-Perdomo O, East TD, Brower R, Morris AH (2015) Standardizing predicted body weight equations for mechanical ventilation tidal volume settings. Chest 148:73-78. https://doi.org/10.1378/chest.14-2843

12. Xiao C, Choi E, Sun J (2018) Opportunities and challenges in developing deep learning models using electronic health records data: a systematic review. J Am Med Inform Assoc 25:1419-1428. https://doi. org/10.1093/jamia/ocy068

13. Friedman JH (2002) Stochastic gradient boosting. Comput Stat Data Anal 38:367-378. https://doi.org/10.1016/S0167-9473(01)00065-2

14. The ARDS Network (2000) Ketoconazole for early treatment of acute lung injury and acute respiratory distress syndrome: a randomized controlled trial. J Am Med Assoc 283:1995-2002

15. Wiedemann HP, Arroliga AC, Komara J et al (2002) Randomized, placebocontrolled trial of lisofylline for early treatment of acute lung injury and acute respiratory distress syndrome. Crit Care Med 30:1-6

16. National Heart, Lung, and Blood Institute Acute Respiratory Distress Syndrome (ARDS) Clinical Trials Network, Matthay MA, Brower RG et al (2011) Randomized, placebo-controlled clinical trial of an aerosolized $\beta_{2}$-agonist for treatment of acute lung injury. Am J Respir Crit Care Med 184:561-568. https://doi.org/10.1164/rccm.201012-20900C

17. Rice TW, Wheeler AP, Thompson BT et al (2011) Enteral omega-3 fatty acid, $\gamma$-linolenic acid, and antioxidant supplementation in acute lung injury. JAMA 306:1574-1581. https://doi.org/10.1001/jama.2011.1435

18. National Heart, Lung, and Blood Institute Acute Respiratory Distress Syndrome (ARDS) Clinical Trials Network, Rice TW, Wheeler AP et al (2012) Initial trophic vs full enteral feeding in patients with acute lung injury: the EDEN randomized trial. JAMA 307:795-803. https://doi.org/10.1001/ jama.2012.137

19. Truwit JD, Bernard GR, Steingrub J et al (2014) Rosuvastatin for sepsisassociated acute respiratory distress syndrome. N Engl J Med 370:21912200. https://doi.org/10.1056/NEJMoa1401520

20. Steinberg KP, Hudson LD, Goodman RB et al (2006) Efficacy and safety of corticosteroids for persistent acute respiratory distress syndrome. N Engl J Med 354:1671-1684. https://doi.org/10.1056/NEJMoa051693

21. National Heart, Lung, and Blood Institute Acute Respiratory Distress Syndrome (ARDS) Clinical Trials Network, Wiedemann HP, Wheeler AP et al (2006) Comparison of two fluid-management strategies in acute lung injury. N Engl J Med 354:2564-2575. https://doi.org/10.1056/NEJMo a062200

22. Zhang Z (2016) Multiple imputation with multivariate imputation by chained equation (MICE) package. Ann Transl Med 4:30. https://doi. org/10.3978/j.issn.2305-5839.2015.12.63

23. DeLong ER, DeLong DM, Clarke-Pearson DL (1988) Comparing the areas under two or more correlated receiver operating characteristic curves: a nonparametric approach. Biometrics 44:837. https://doi. org/10.2307/2531595 
24. ARDS Definition Task Force, Ranieri VM, Rubenfeld GD et al (2012) Acute respiratory distress syndrome: the Berlin definition. JAMA 307:2526-2533

25. Pham T, Serpa Neto A, Pelosi P et al (2019) Outcomes of patients presenting with mild acute respiratory distress syndrome: insights from the LUNG SAFE study. Anesthesiology 130:263-283. https://doi.org/10.1097/ ALN.0000000000002508

26. Villar J, Pérez-Méndez L, Kacmarek RM (2016) The Berlin definition met our needs: no. Intensive Care Med 42:648-650. https://doi.org/10.1007/ s00134-016-4242-6

27. Wang C, Wang X, Chi C et al (2016) Lung ventilation strategies for acute respiratory distress syndrome: a systematic review and network metaanalysis. Sci Rep 6:22855. https://doi.org/10.1038/srep22855

28. Fan E, Brodie D, Slutsky AS (2018) Acute respiratory distress syndrome: advances in diagnosis and treatment. JAMA 319:698-710. https://doi. org/10.1001/jama.2017.21907

29. Zhang Z, Hu X, Zhang $X$ et al (2015) Lung protective ventilation in patients undergoing major surgery: a systematic review incorporating a Bayesian approach. BMJ Open 5:e007473. https://doi.org/10.1136/bmjop en-2014-007473

30. Patel JM, Baker R, Yeung J et al (2016) Intra-operative adherence to lungprotective ventilation: a prospective observational study. Perioper Med (Lond) 5:8. https://doi.org/10.1186/s13741-016-0033-4

31. Villar J, Martín-Rodríguez C, Domínguez-Berrot AM et al (2017) A quantile analysis of plateau and driving pressures: effects on mortality in patients with acute respiratory distress syndrome receiving lung-protective ventilation. Crit Care Med 45:843-850. https://doi.org/10.1097/CCM.00000 00000002330
32. Villar J, Ambrós A, Soler JA et al (2016) Age, $\mathrm{PaO}_{2} / \mathrm{FIO}_{2}$, and plateau pressure score: a proposal for a simple outcome score in patients with the acute respiratory distress syndrome. Crit Care Med 44:1361-1369. https:// doi.org/10.1097/CCM.0000000000001653

33. Park M, Ahn HJ, Kim JA et al (2019) Driving pressure during thoracic surgery: a randomized clinical trial. Anesthesiology. https://doi.org/10.1097/ aln.0000000000002600

34. Laffey JG, Bellani G, Pham T et al (2016) Potentially modifiable factors contributing to outcome from acute respiratory distress syndrome: the LUNG SAFE study. Intensive Care Med 42:1865-1876. https://doi.org/10.1007/ s00134-016-4571-5

35. Hotchkiss JR, Blanch L, Murias G et al (2000) Effects of decreased respiratory frequency on ventilator-induced lung injury. Am J Respir Crit Care Med 161:463-468. https://doi.org/10.1164/ajrccm.161.2.9811008

36. Maeda Y, Fujino Y, Uchiyama A et al (2004) Effects of peak inspiratory flow on development of ventilator-induced lung injury in rabbits. Anesthesiology 101:722-728

37. Fujita Y, Fujino Y, Uchiyama A et al (2007) High peak inspiratory flow can aggravate ventilator-induced lung injury in rabbits. Med Sci Monit 13:BR95-BR100

38. Zhang Z, Spieth PM, Chiumello D et al (2019) Declining mortality in patients with acute respiratory distress syndrome: an analysis of the acute respiratory distress syndrome network trials. Crit Care Med 47:315-323. https://doi.org/10.1097/CCM.0000000000003499 